\title{
MAPEAMENTO DO RECURSOS HÍDRICOS DE SUB-BACIAS HIDROGRÁFICAS DO PONTAL DO PARANAPANEMA - SP.
}

\author{
WATER RESOURCES MAPPING OF BASINS IN THE PONTAL DO \\ PARANAPANEMA - SP.
}

Nangly Xavier Ribeiro ${ }^{1}$, Lucas Prado Osco', Ana Paula Ramos ${ }^{1}$, Antonio Cezar Leal ${ }^{2}$

${ }^{1}$ Universidade do Oeste Paulista - UNOESTE, Curso de Engenheiro Ambiental,
Mestrado em Meio Ambiente e Desenvolvimento Regional, Presidente Prudente - SP.
${ }^{2}$ Universidade Estadual Paulista - UNESP, Departamento de Geografia, Presidente
Prudente, SP.
E-mail: anaramos@unoeste.br

RESUMO - Na atual crise hídrica, uma necessidade ainda mais em evidência é o eficaz gerenciamento e preservação da qualidade das águas. Uma bacia hidrográfica deve ser compreendida enquanto uma unidade de planejamento ambiental. Contudo, a realização de planejamento ambiental de bacias requer a elaboração de diferentes produtos cartográficos, os quais oferecem suporte aos processos de tomada de decisão. O objetivo deste trabalho é realizar o mapeamento dos recursos hídricos de sub-bacias hidrográficas da Unidade de Gerenciamento de Recursos Hídricos do Pontal do Paranapanema (UGRHI-22). Este trabalho contribui para o procedimento metodológico que deve ser realizado quando o intuito é executar o planejamento ambiental de bacias. O método empregado consistiu no uso de técnicas de geoprocessamento e de interpretação de ortofotografias digitais de alta resolução. A partir disso, fez-se o mapeamento, na escala 1:3.000, dos cursos d'águas e das áreas úmidas de três sub - bacias da UGRHI-22. Os resultados possibilitam compreender a distribuição dos recursos hídricos em cada uma das bacias de estudo. Conclui-se que a densidade de drenagem das subbacias é de média a alta, e que há escassa cobertura vegetal, especialmente nas áreas de preservação permanente ao longo dos cursos d'água.

Palavras-chave: Bacia Hidrográfica; Mapeamento; Recursos Hídricos; Planejamento Ambiental.

Recebido em: 19/08/2015 Revisado em: 08/09/2015 Aprovado em: 10/09/2015
ABSTRACT - In the current water crisis, a need further highlight is the effective management and preservation of water quality. A watershed must be understood as a unit of environmental planning. However, the realization of environmental planning basins required to produce different cartographic products, which support the decision-making processes. The objective of this work is to perform the mapping of water resources in watershed of the Water Resources Management 
Unit of the Pontal do Paranapanema (UGRHI-22). This work contributes to the methodological procedure that should be performed when the intention is to run environmental planning watershed. The method employed was the use of geoprocessing and interpretation of digital orthophotos of high resolution. From this it became the mapping in scale 1: 3,000, the water courses and wetlands of three watershed UGRHI-22. The results make it possible to understand the distribution of water resources in each of the study watershed. It concludes that the watershed drainage density is medium to high, and there is little vegetation cover, especially in the areas of permanent preservation over the waterways.

Keywords: Watershed Mapping; Water Resources; Environmental Planning. 


\section{INTRODUÇÃO}

A falta de água tem sido um tema de estudo de longos anos em diferentes países, incluindo-se o Brasil. Isto se deve a vários fatores, como a poluição dos corpos d'água, o manejo inadequado, entre outros. Segundo Albuquerque (2012 apud BOTELHO, 1999), a área drenada por um rio principal e seus afluentes, que pode ser delimitada a partir da interpretação do relevo em mapas ou cartas planialtimétricas, pelos seus divisores de água, é denominada bacia hidrográfica. Para manter os rios e a qualidade das águas, tanto superficial como subterrâneas, nas bacias hidrográficas torna-se necessário desenvolver o planejamento ambiental dessas bacias.

O planejamento ambiental tem como objetivo proteger os ambientes naturais e permitir o uso sustentável dos recursos naturais disponíveis para o ser humano (SANTOS, 2014). Dentre as etapas que constituem o planejamento ambiental de bacias hidrográficas, tem-se a produção cartográfica da área, que possibilita compreender a distribuição dos corpos e cursos d'água de uma bacia hidrográfica. Assim, a função dos produtos cartográficos é de auxiliar nas análises e nos processos de tomada de decisão envolvidos no planejamento ambiental de uma bacia.
O objetivo deste trabalho é realizar o mapeamento dos recursos hídricos de subbacias hidrográficas da Unidade de Gerenciamento de Recursos Hídricos do Pontal do Paranapanema (UGRHI-22). Este trabalho contribui para o procedimento metodológico que deve ser realizado quando o intuito é executar o planejamento ambiental de bacias hidrográficas. A UGRHI22 é subdividida em IV unidades de gerenciamento: I, II, III e IV. As sub-bacias em estudo, pertencem a IV unidade, sendo as quais: Ribeirão dos Patos, Córrego dos Patinhos e Córrego da Cachoeira.

Cabe ressaltar que 0 presente trabalho é parte do Projeto Temático “Mapeamento e Análise do Território do Agrohidronegócio Canavieiro no Pontal do Paranapanema-São Paulo-Brasil: Relações de trabalho, conflitos e formas de uso da terra e da água, e a saúde ambiental", coordenado pelo Prof. Dr. Antonio Thomaz Júnior, processo FAPESP (Processo: 2012/23959-9). Assim, o mapeamento vem sendo desenvolvido em parceria entre a Universidade Estadual Paulista (UNESP), Faculdade de Ciências e Tecnologia, campus de Presidente Prudente, vinculados ao Grupo de Pesquisa em Gestão Ambiental e Dinâmica Socioespacial (GADIS), sob a orientação do Prof. Dr. Antonio Cezar Leal (UNESP), e a Universidade do Oeste Paulista (UNOESTE), por meio do Núcleo de Estudos Ambientais e 
Geoprocessamento (NEAGEO) e do Programa de Mestrado em Meio Ambiente e Desenvolvendo Regional/MMADRE.

\section{METODOLOGIA}

O método empregado consistiu no uso de técnicas de geoprocessamento e de interpretação visual de ortofotografias digitais de alta resolução. A partir disso, fezse o mapeamento, na escala 1:3.000, dos cursos d'águas e das áreas úmidas de três sub-bacias da UGRHI-22. Para tanto, a elaboração do presente trabalho foi iniciada com o levantamento bibliográfico sobre o tema planejamento ambiental. Desta forma, fundamentou-se nas obras de Albuquerque (2012), Botelho (1999), Santos (2004) e Hiruma e Ponçano (1994). Na sequência, fezse a caracterização da localização geográfica das áreas de estudos conforme apresentado na Figura 1. A Unidade de Gerenciamento de Recursos Hídricos do Pontal do Paranapanema está totalmente inserida na província geomorfológica, denominada Planalto Ocidental Paulista. Um total de 26 municípios compõe a UGRHI-22, de forma integral e(ou) parcialmente (CBH-PP apud CTPI, 1999).

Figura 1. Localização Geográfica das Áreas de Estudo.

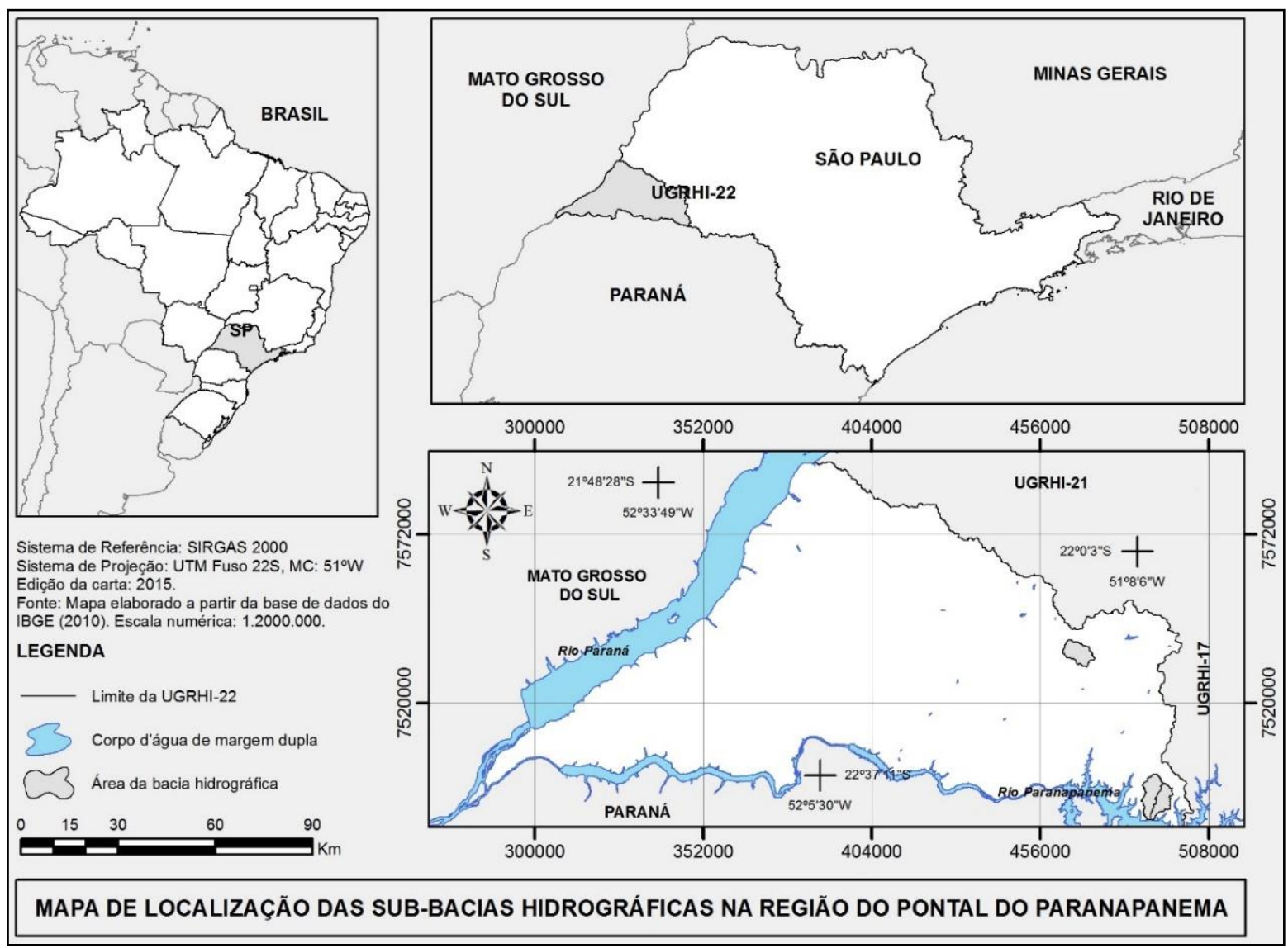


Para o mapeamento dos recursos hídricos das sub-bacias em estudo - Ribeirão dos Patos, Córrego dos Patinhos e Córrego da Cachoeira, utilizou-se ortofotografias digitais fornecidas pela EMPLASA (2011). Tais ortofotos foram geradas com GSD (Ground Sample Distance) de 1 metro de resolução espacial, e são classificadas com Padrão de Exatidão Cartográfica (PEC) classe $A$, na escala 1: 25000 (EMPLASA, 2011). A vetorização dos recursos hídricos foi realizada utilizando o Sistema de Informação Geográfica (SIG) da empresa ESRI, o ArcGIS 10.2. Realizou-se a vetorização dos cursos d'água e das áreas úmidas por meio da interpretação visual das ortofotos digitais, utilizando a primitiva de polígonos, e a escala de visualização de 1:3.000. Para a interpretação das imagens, utilizou-se uma chave de interpretação elaborada para a região de estudo.

\section{RESULTADOS}

Como resultados deste trabalho, temse o mapeamento da drenagem e das áreas úmidas presentes nas três sub-bacias localizadas na unidade de planejamento IV da UGRHI-22. As duas primeiras sub-bacias, ribeirão dos Patos e córrego dos Patinhos estão localizadas uma ao lado da outra e possuem área de $48 \mathrm{Km}^{2}$ e $38 \mathrm{Km}^{2}$, respectivamente. A Figura 2 apresenta o resultado do mapeamento dos recursos hídricos nesta bacia hidrográfica. Nota-se (Figura 2) que as sub- bacias possuem densidade de drenagem moderada devido às poucas ramificações. A classificação de número de ordem de ambas sub-bacias é 3 (três), o que indica chances moderadas de enchentes na área.

A terceira sub-bacia é a do córrego da cachoeira e possui uma área de $52 \mathrm{~km}^{2}$ de extensão. A Figura 3 ilustra o resultado do mapeamento dos recursos hídricos dessa bacia. A sub - bacia córrego da Cachoeira, possui uma densidade de drenagem elevada sendo a classificação de número de ordem desta sub - bacia 5 (cinco), obtendo grandes chances de enchentes. 
Figura 2. Distribuição dos recursos hídricos das sub-bacias hidrográficas Ribeirão do Patos e Córrego dos Patinhos

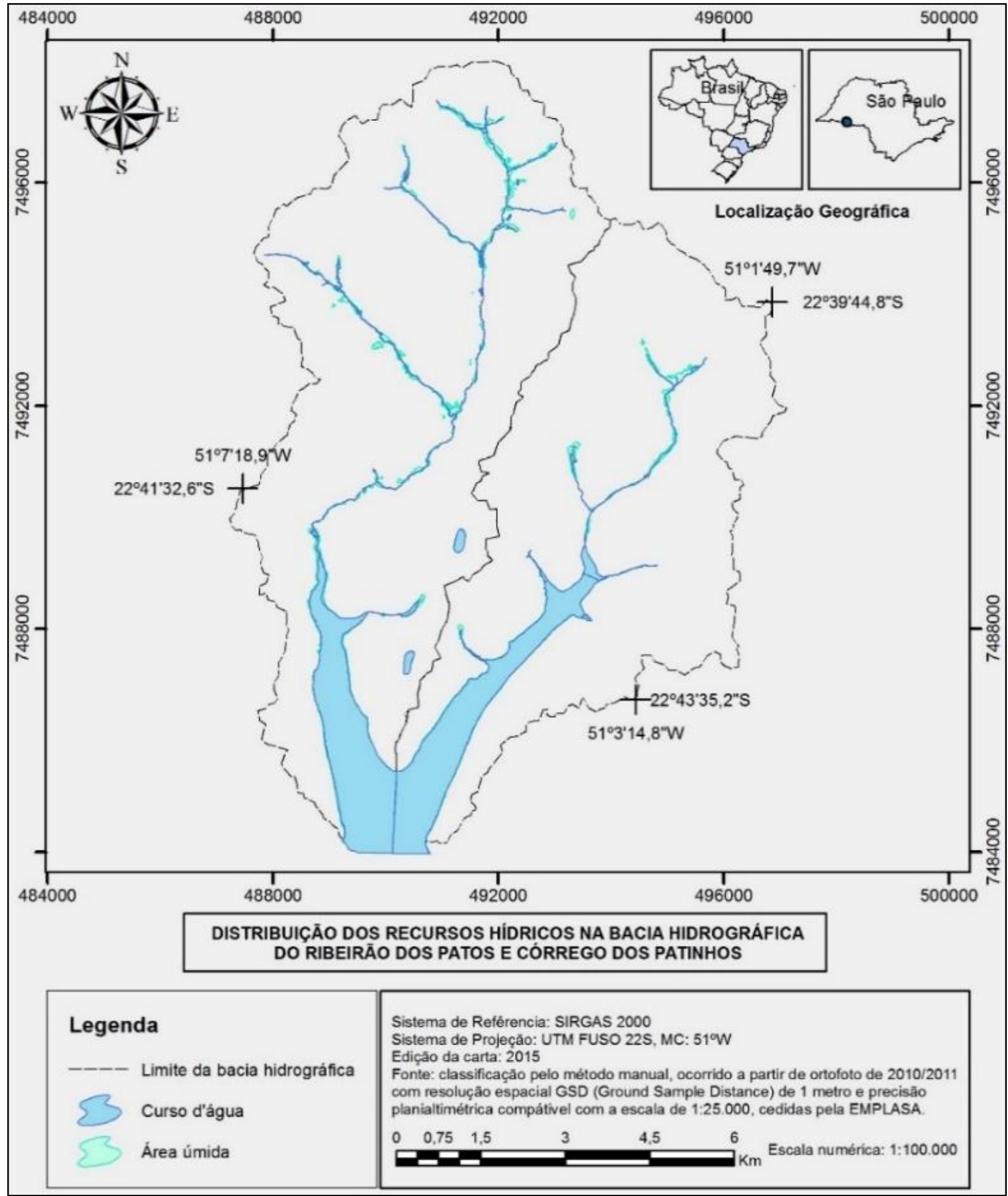


Figura 3. Recursos hídricos da sub-bacia Hidrográficas Córrego da Cachoeira

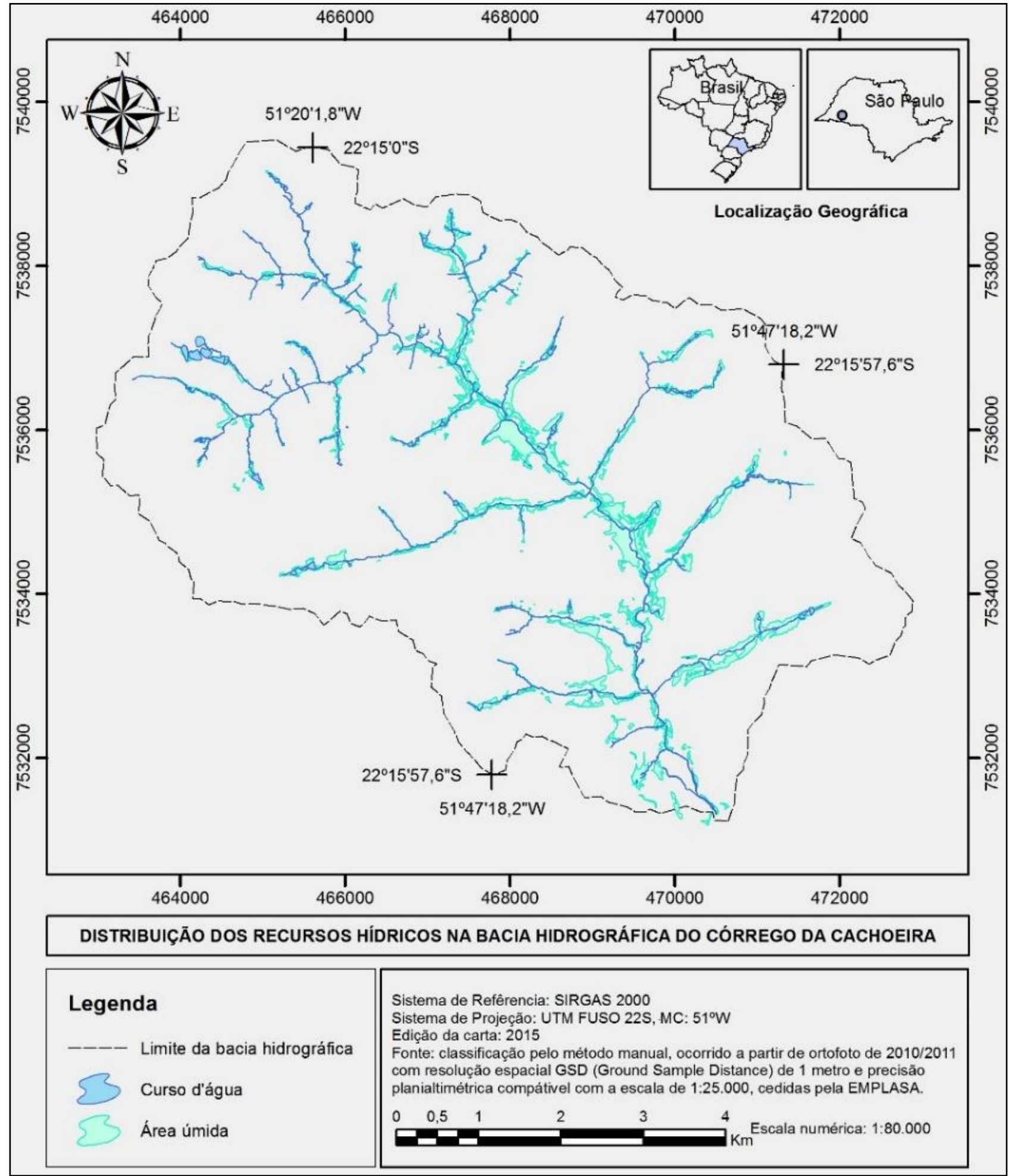

\section{DISCUSSÃO}

A densidade de drenagem foi definida por Horton em 1945 e é considerada um dos mais importantes parâmetros da análise morfométrica de bacias hidrográficas (HIRUMA; PONÇANO, 1994). A área de drenagem de uma bacia hidrográfica é toda área geográfica onde a precipitação escoa para o mesmo ponto de saída. A densidade 
de drenagem elevada ocorre em regiões de solo impermeável, de declividades elevadas, fácil erosão e pouca cobertura vegetal. Dados estes que corroboram com as três bacias em estudo, as quais possuem densidades de moderada a elevada. $\mathrm{Na}$ vetorização dos recursos hídricos, pode-se identificar a escassa cobertura vegetal, principalmente, a falta de vegetação nas áreas de preservação permanente (APP's).

\section{CONCLUSÃO}

Este trabalho propôs a realização do mapeamento dos recursos hídricos de três sub-bacias hidrográficas da Unidade de Gerenciamento de Recursos Hídricos do Pontal do Paranapanema, a UGRHI-22. Conclui-se que as sub-bacias do Ribeirão dos Patos e do Córrego dos Patinhos sofrem riscos de enchentes devido a densidade de drenagem moderada, além de possuírem características de falta de cobertura vegetal e suscetibilidade a erosões. Quanto à subbacia Córrego da Cachoeira, esta apresenta indícios de riscos de enchentes, em razão da densidade de drenagem elevada, e também possui características de pouca cobertura vegetal. Evidencia-se com este trabalho a necessidade de um melhor planejamento ambiental dessas sub-bacias da UGRHI-22, que contribua para a preservação e conservação dos recursos hídricos, os quais são essenciais à vida.
Os resultados da pesquisa serão disponibilizados aos órgãos de gestão ambiental e ao Comitê de Bacias Hidrográficas, para subsidiar à atividade de planejamento ambiental dessas sub-bacias. Além disso, sugere-se a realização de outros estudos, como o de uso e ocupação do solo, visando principalmente a melhor compreensão das fragilidades e potencialidades dessas sub-bacias hidrográficas do Pontal do Paranapanema.

\section{REFERÊNCIAS}

ALBUQUERQUE, A. R. C. Bacia hidrográfica: unidade de planejamento ambiental. Revista Geonorte, Edição Especial, v.4, n.4, p.201209, 2012.

BOTELHO, R. G. M; GUERRA, A.J.T.; SILVA, A. S.da. Erosão e conservação dos solos: conceitos, temas e aplicações.; Rio de Janeiro: Bertand Brasil, 1999. 340p.

BOTELHO, R. G. M; SILVA, A. S.; VITTE, A. C. Bacia hidrográfica e qualidade ambiental. In: VITTE, A. C.; GUERRA, A. J. T. (org.). Reflexões sobre a geografia física no Brasil. Rio de Janeiro: Bertrand Brasil, 2004. cap. 6.

CTPI - Cooperativa de Serviços, Pesquisas Tecnológicas e Industriais. Diagnóstico da situação dos recursos hídricos da UGRHI 22. Pontal do Paranapanema: Relatório Zero. São Paulo: CPTI, 1999. CD-ROM.

EMPLASA - Empresa Paulista de Planejamento Metropolitano. Projeto Mapeia São Paulo - Informações Técnicas (2011). Disponível em: $<$ http://www.mapeiasp.sp.gov.br/Images/Infor macoes_Tecnicas.pdf $\geq$. Acesso em: 07 ago. 2015. 
HIRUMA, S. T; PONÇANO, W. L. Densidade de drenagem e sua relação com fatores feomorfopedológicos na área do alto rio Pardo, SP e MG. Rev. IG. São Paulo, v.15, n.112, p.49-57, jan./dez. 1994.

SANTOS, R. F. Planejamento ambiental:

teoria e prática. São Paulo: Oficina de textos, 2004.

UFRCS. Centro de Processamento de Dados.

ArGis. 2015. Disponível em:

$<$ Http://www.ufrgs.br/cpd/servicos/computa dores-e-aplicativos/softwaresdisponiveis/software-licenciado-arcgis>.

Acesso em: 21 jul. 2015. 